\title{
Observed effects of star-planet interaction
}

\author{
Scott J. Wolk ${ }^{1}$ and Ignazio Pillitteri ${ }^{1,2}$ and Katja Poppenhaeger ${ }^{1}$ \\ ${ }^{1}$ Harvard Smithsonian Center for Astrophysics, 60 Garden Street, \\ Cambridge MA 02138, USA \\ email: swolk@cfa.harvard.edu, kpoppen@cfa.harvard.edu \\ ${ }^{2}$ Osservatorio Astronomico di Palermo, piazza del Parlamento 1, I-90134 Palermo, Italy \\ email: pilli@astropa.unipa.it
}

\begin{abstract}
Since soon after the discovery of hot Jupiters, it had been suspected that interaction of these massive bodies with their host stars could give rise to observable signals. We discuss the observational evidence for star-planet interactions (SPI) of tidal and magnetic origin observed in X-rays and FUV. Hot Jupiters can significantly impact the activity of their host stars through tidal and magnetic interaction, leading to either increased or decreased stellar activity - depending on the internal structure of the host star and the properties of the hosted planet. In HD 189733, X-ray and FUV flares are preferentially in a very restricted range of planetary phases. Matsakos et al. (2015) show, using MHD simulations, planetary gas can be liberated, forming a stream of material that gets compressed and accretes onto the star with a phase lag of 70-90 degrees. This scenario explains many features observed both in X-rays and the FUV (Pillitteri et al. 2015). On the other hand, WASP-18 - an F6 star with a massive hot Jupiter, shows no signs of activity in X-rays or UV. Several age indicators (isochrone fitting, Li abundance $)$ point to a young age $(\sim 0.5--1.0 \mathrm{Gyr})$ and thus significant activity was expected. In this system, tidal SPI between the star and the very close-in and massive planet appears to destroy the formation of magnetic dynamo and thus nullify the stellar activity.
\end{abstract}

Keywords. X-rays: stars, magnetic fields, exoplanets, interactions

\section{Introduction}

Over the last decade, the discovery of exoplanets has fundamentally changed our perception of the universe and humanity's place within it. The role of X-rays in the study of exoplanets is subtle, but recent work indicates exoplanets, especially hot Jupiter systems, are unique X-ray environments and the impact of X-rays may be significant for the evolution of the system. The effects can work several ways; the intense high energy flux alters the thermal budget of the upper atmosphere of planet, the angular momentum and magnetic field of the planet can induce more activity on the star and the enhanced X-rays are absorbed by the transiting planet, which, in turn, act as a probe of the planetary upper atmosphere. In addition, an overall enhancement of the stellar host activity can significantly influence the chemistry of any additional planet in the habitable zone of the same star and thus the evolution of life in the system.

As first noted by Lammer et al. (2003), inclusion of stellar X-ray and EUV flux in irradiance calculations leads to energy-limited escape and atmospheric expansion not found in models incorporating stellar UV/optical/IR insulation alone. The increased mass loss rates are of order $10^{12} \mathrm{~g} \mathrm{~s}^{-1}$, implying hydrogen-rich exoplanets may evaporate and shrink to levels at which heavier atmospheric constituents may prevent hydrodynamic escape. The generation of an exosphere due to local X-ray luminosity has been directly detected in the case of the planets HD 209458b and HD 189733b. Absorption by atmospheric gas has been used to probe the layer where the gas escapes in the upper atmosphere 
(e.g. Vidal-Madjar 2003, Ballester et al. 2007 Poppenhaeger et al.2013). Modeling by Penz et al. (2008) shows evolution of close-in exoplanets strongly depends on the detailed X-ray luminosity history of their host stars. Stars located at the high end of the X-ray luminosity distribution evaporate most of their planets' atmospheres within $0.05 \mathrm{AU}$, while a significant fraction of planets can survive if exposed to a moderate X-ray luminosity. At lower X-ray luminosities, they find that the mass loss is negligible for hydrogen-rich Jupiter-mass planets at orbits $>0.02$ AU, while Neptune-mass planets are influenced up to $0.05 \mathrm{AU}$ (see also Murray-Clay et al.2009).

Star-planet Interactions (SPI) not only proceed from star to the planet, but theoretical arguments demonstrate that hot Jupiters (HJs; planets with masses approximately equal to or greater than Jupiter with an orbital semi-major axis of less than 0.5 AU) can also influence their host by means of tides and magnetic fields. Cuntz et al.(2000) showed that energy generation due to tidal perturbations is proportional to $a^{-3}$. HJs induce tidal bulges on the host star; cool stars can dissipate the energy contained in the bulges much more effectively than hot stars due to turbulent eddies in the convective envelopes (Zahn 2008). The energy released via reconnection during an interaction of the planetary magnetosphere with the stellar magnetic field is estimated as $F_{\text {int }} \propto B_{*} \times B_{P} v_{\text {rel }} a_{P}^{n}$ (with $n \sim-3$; Saar et al. 2004).

The focus of this presentation is to look at evidence for signs of feedback between the stars and planets which may enhance or nullify the high energy irradiance which is crucial for evolution of planets as well as our estimation of their habitability. For our purposes, star-planet interaction (SPI) is driven by magnetic interaction between the stellar and planetary magnetic fields, or by tidal interaction (Cuntz et al., 2000). Both effects strongly depend on the planet-star separation, which is directly measurable. But the observation result is also a function of the intensity and topology of the magnetic fields, and the internal structure of the star - which are less apparent.

\section{Observations}

For the purposes of this contribution we will focus on observations of three systems with HJs. The second and third cases are edge cases representing a highly eccentric system and a high mass planet respectively. The first case, on the other hand, is the gold standard of HJs.

\section{1. $H D 189733$}

HD 189733 is one of the best studied systems with a transiting HJ. It is in a binary system with a quite inactive and old $(\tau>5$ Gyr) M4 secondary star. This system was originally thought to be about $600 \mathrm{Myr}$, based on a relatively high activity level of the primary which led to the assumption it was a Hyades member. Pillitteri et al.(2010) noted they did not detect the M4 companion in the XMM image although it should have been bright enough if it were indeed $600 \mathrm{Myr}$. They speculated that the activity in the primary was enhanced by the interaction with the exoplanet. Indeed, when Poppenhaeger, Schmitt \& Wolk (2013) detected the secondary star with the Chandra X-ray Observatory, they found an activity level consistent with an age of about 5 Gyr (see also the presentation at this symposium by Poppenhaeger).

In addition to the age anomaly, Pillitteri et al.(2014) discuss three eclipse observations of HD 189733b (Fig. 1). Each time they noted a significant flare within hours after the eclipse. They speculated this was due to a hot spot forward phased from the sub planetary point by about $90^{\circ}$, consistent with analytic predictions by Lanza (2008). Using HST, Pillitteri et al.(2014) acquired high quality COS spectra in the wavelength 


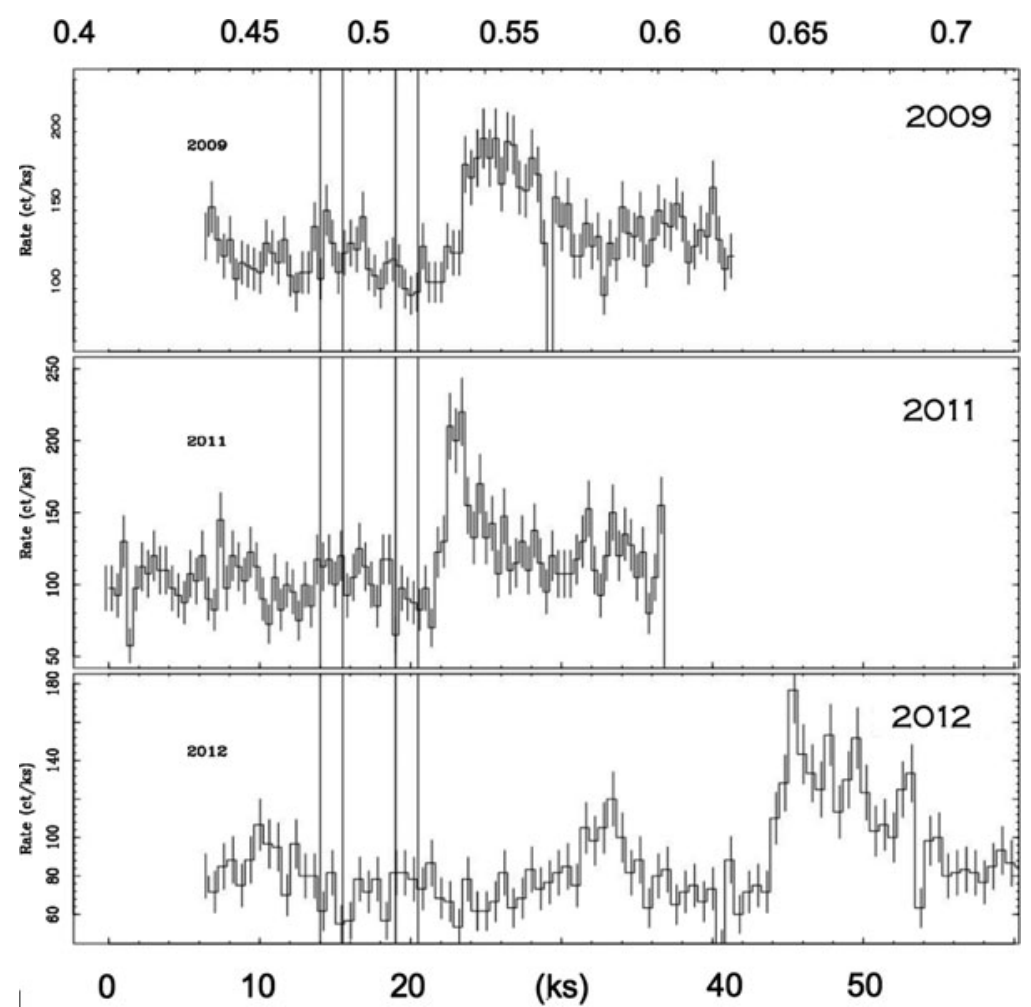

Figure 1. Light curves for the XMM-Newton EPIC/PN observations of three eclipses of HD 189733b. The top $\mathrm{X}$-axis indicates phase. The bottom $\mathrm{X}$-axis indicates time (normalized to the phase start of the 2011 observation). First through fourth contacts are indicated by the vertical lines. In all cases, the quiescent count rates are within $20 \%$ of $100 \mathrm{cnt} / \mathrm{ks}$.

range 1150-1450 A. Again, flares were observed just after the eclipse. They found two episodes of strong variability of the line fluxes of ions of $\mathrm{Si}, \mathrm{C}$, and $\mathrm{N}$ that had not been observed in planetary transits. The details of the flares were consistent with an MHD model (Matsakos et al. 2015). The flow morphology in the model provides a natural explanation of the FUV line and X-ray variability of HD 189733. Specifically, the plasma is liberated from the upper atmosphere of the planet and funneled by the magnetized stellar wind in an almost radial trajectory close to the star. The flow forms a knee structure that consists of hot and dense plasma which then accretes in a region of the star fixed with the synodic phase (Fig. 2).

On the other hand, the X-ray flare observed in 2012, had aspects of reverberation. During the decay of the flare, three successively smaller peaks are observed separated by about $4 \mathrm{ks}$. This appears to have been a damped magneto acoustic oscillation in a flaring loop (e.g. Mitra-Kraev et al.2005). In such loop the change of the intensity of the successive peaks can be described as:

$$
\frac{\Delta I}{I} \sim \frac{4 \pi n k_{B} T}{B^{2}}
$$

Since spectral fits to the XMM spectra can be used to determine the temperature and the density, this formulation can be used to measure the magnetic field if magneto acoustic oscillation is the cause of the flare structure. In this case, the derived B field $(\sim 40 \mathrm{G})$ is consistent with results of spectropolarimetry (Fares et al. 2010). With the reverberation 

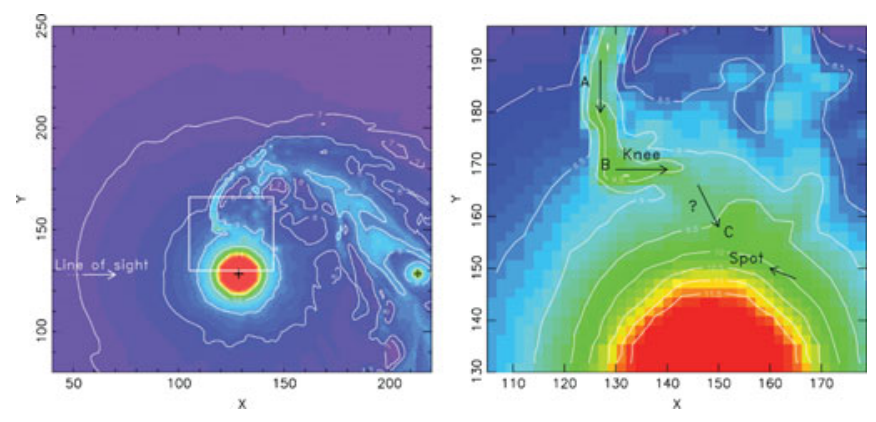

Figure 2. Particle density contours of an MHD simulation that models star-planet interactions between a HJ and its host (polar view). The star rotates counterclockwise, and the planet orbits the star along the same direction. The two "+" symbols shown on the left panel indicate the location of the star (red disk) and the planet (green disk). Right panel: a close-up of the impact region, where the motion of the accreting plasma is marked with arrows. Specifically, the shocked plasma is funneled by the magnetized stellar wind in an almost radial trajectory close to the star (A), forms a "knee" structure that consists of hot and dense plasma (B), and then accretes in a spot ahead of the orbital phase $(\mathrm{C})$. The precise details of accretion are not investigated by the simulation (zone marked with ?). The knee (B) of the stream and the active spot upon impact on the surface $(\mathrm{C})$ are the main sites of production of the enhanced flux observed in the FUV and X-ray bands and phased with the orbital motion (from Pillitteri et al. 2015).

hypothesis thus supported, the length of the loop can be calculated by simple argument about the sound speed, a function of the density, and the travel time (the time between the successive peaks). The result is a loop length of about $4 \mathrm{R}_{*}$ indicating a flare covering half the distance to the planet. While there is no evidence that the flare is actually directed to the planet, the result is reminiscent of Favata et al.(2005) and McCleary \& Wolk (2011). Both groups found that for flares in PMS stars, long length flares only occurred in cases where the star was surrounded by a disk. Both groups concluded that the flares stretched from the star to the disk in these cases. By analogy the magnetic field of the planet could be acting as a footpoint for occasional, massive, flares. Such flares would have significant impacts on the planet, ionizing material in its upper atmosphere. Since it appears to be tidal interaction between the star and the planet which is the prima facia cause of the enhanced stellar field, planetary orbital energy is the ultimate source of the flare that scorches the planet's atmosphere.

\section{2. $H D 17156$}

In addition to HJs close to their host stars, eccentric systems - in which the Jupiter is "hot" only a fraction of the time - should be a good test bed for the existence of SPI. The prediction is that SPI effects only occur when the star and planet are close. One candidate for such a test is HD 17156.

HD 17156 is a G0 star with a HJ in a 21 day orbit (Barbieri et al. 2007). The eccentricity of the orbit is 0.68 . The planet reaches a minimum separation of $\sim 15 \mathrm{R}_{*}$. XMM-Newton observed HD 17156 when the planet was at the periastron and a second time, when the planet was more distant. HD 17156 was not detected by XMM when the two were separated. However, just after periastron passage, there was a marked rise in the X-ray luminosity with a corresponding rise in the chromospheric activity. Maggio et al.(2015) suggest that this could have been either due a magnetic reconnection and or flaring activity when the planet was at its minimum separation or due to material stripped from the planet and falling onto the star. The excess of X-rays has a soft spectrum and could favor the tidal stripping hypothesis. 

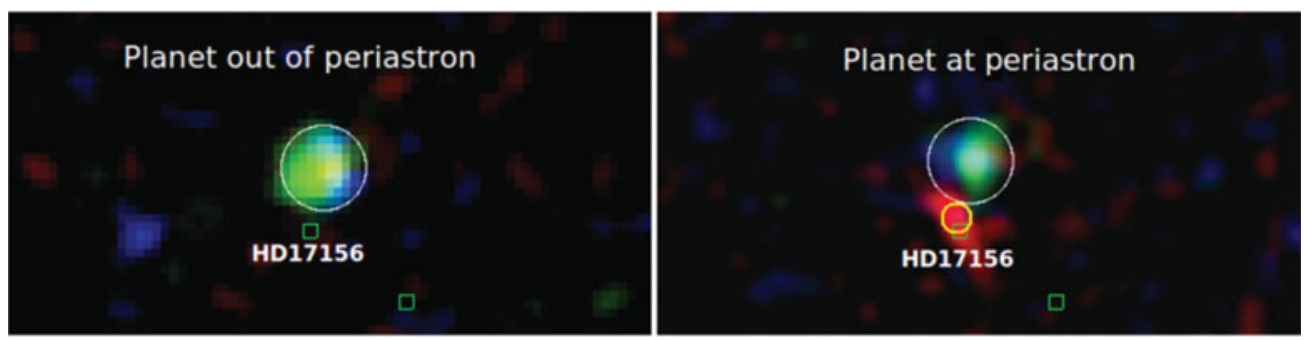

Figure 3. X-ray images of HD 17156 taken far from the planetary periastron (left panel) and near the periastron (right panel). The image is colored by photon energy (red $=0.3-1.0 \mathrm{keV}$, green $=1.0-2.5 \mathrm{keV}$, blue=2.5-5.0 keV). Smoothing is applied to the images, with a Gaussian $\sigma=4^{\prime \prime}$. Positions of the only two objects in the SIMBAD catalog are shown with small squares. Circle sizes indicate the wavelet detection scales of HD 17156 and of an unrelated background object with a harder spectrum (From Maggio et al.2015).

\subsection{WASP-18}

WASP-18 (F6V) has one of the fastest orbiting and most massive HJs. The planet has a mass of over $10 \mathrm{M}_{J u p}$ and the period is about 22 hours. The estimate of the age for this system is about 600 Myrs. This is based on both isochrone fitting and the strong $\mathrm{Li}$ absorption. Indeed, the observed $\mathrm{Li}$ strength is a near perfect match for Hyades and Beehive cluster stars of similar temperature. The expected X-ray luminosity from such a star should be at least $\log \mathrm{L}_{X} \sim 28.5$. This is nearly the same as observed from HD 189733 and a priori might indicate strong magnetic re-connection events.

In fact, exactly the opposite is seen. No X-rays were detected with a flux limit of $\log \mathrm{L}_{X} \sim 26.5$. Fossati et al.(2014) asserted that the lack of chromospheric activity detected from WASP-18 (and WASP-12) may have been due to local absorption of UV by atmospheric material stripped off of the planet by tidal forces. We find this unlikely for several reasons: First, given the high mass of WASP-18b, atmospheric stripping should be 100 times less effective in this case than HD 189733. Second, the calcium absorption line is observed. It is only the emission reversal which is not observed. Third, no X-rays are detected at all, not even high energy X-rays which might be expected from the Planck tail of a $1 \mathrm{keV}$ thermal distribution. Pillitteri et al.(2014) conclude the the star is X-ray dark and suggest tidal interaction with the planet must have a role in destroying the dynamo efficiency and the overall activity of the star. Based on the formulae given in Cuntz et al.(2000), tides on WASP-18 are the largest of any known exoplanet host, of order of $500 \mathrm{~km}$ because of planet proximity and its mass. During the Symposium, T. Ayers pointed out that a complete break down in convection was not possible as this is key to energy transport in the star. The argument by Pillitteri et al.(2014) is that the tidal wave disrupts the shear layer at the top of the convection zone. This prevents the build-up and concentration of magnetic energy close to the surface. Meanwhile the convective thermal transport from the core to near the surface is free to occur. Near the surface, radiative cooling processes dominate.

\section{Conclusions}

Over the past five years there has been a lot of case specific evidence gathered that stars can interact with their HJ companions via tides and magnetic fields. The level of confidence in each kind of evidence varies.

In an earlier presentation, Poppenhaeger (Sym. 320.11.04) discussed using binaries to test for activity induced by HJs (Poppenhager \& Wolk 2014). She presented at least 3 
examples of binaries in which the HJ host had an activity level incompatible with the age indicated by the activity level of the non-hot Jupiter host. She concluded that HJs spin-up stars when the HJ host has a large convective zone. All the observed data are consistent with this hypothesis and it is strongest direct evidence of SPI. The corollary to this is uncertainty in using activity to age date stars with close in planets.

Prior to eclipse observations, analytical calculations predicted the existence of an active region on HD 189733 forward phased by about $90^{\circ}$ from the sub-planetery point. This would imply enhanced activity between phases 0.5 and 1.0. The propensity for the star to flare between phase 0.55 and 0.65 has been taken as evidence of the active region, perhaps being observed over the limb. In total there have been 5 high energy flares observed in four observations (including the FUV observations). The significance of this is unclear and fraught with over interpretation. So while the result is suspicious it is not yet compelling.

The two remaining pieces of evidence, the long length flare in HD 189733 and enhanced activity and periapses for HD 17156 are tantalizing. On the other hand, each of these has only been seen to occur once. Until additional flares are seen in HD 17156 or another eccentric system in periapses passage this will stand as a one-time occurrence. The expected frequency for such long length flares is unknown, but it appears long. In $850 \mathrm{ks}$ observing the ONC only 25 such flares were observed among about 400 disked stars (Favata et al.2005).

Statistical evidence is a different story. Several groups (Kayshap et al.2008, Poppenhaeger et al.2011 and Miller et al.2015) have looked for statistical evidence of SPI, with mixed results. The problem is that many parameters effect the eventual outcome of the star-planet interaction. Mass and distance ratios are the obvious parameters, but depth of convection and relative field orientation and strength are clearly others. The cautionary tale of WASP-18 indicates that tidal forces can be constructive or destructive when it comes to enhancing stellar activity. Any statistical test needs to account properly for outliers. Both the high and low outliers may be the result of SPI while the median and mean may not be very affected. The coming few years should prove very interesting as we continue to gather evidence.

\section{References}

Barbieri, M., et al. 2007, A\& A, 476, L13

Favata, F., Flaccomio, E., \& Reale, F., et al. 2005, ApJS, 160, 469

Fares, R., et al. 2010, MNRAS, 406, 409

Fossati, L., Ayres, T. R., \& Haswell, et al. 2014, APSS, 354, 21

Kashyap, V. L., Drake, J. J., \& Saar, S. H. 2008, ApJ, 687, 1339

Lanza, A. F. 2008, A\& $A$, 487, 1163

Maggio, A., et al. 2015, ApJL, 811, L2

McCleary, J. E. \& Wolk, S. J. 2011, AJ, 141, 201

Miller, B. P., Gallo, E., Wright, J. T., \& Pearson, E. G. 2015, ApJ, 799, 163

Mitra-Kraev, U., Harra, L. K., Williams, D. R., \& Kraev, E. 2005, A\&\&A, 436, 1041

Murray-Clay, R. A., Chiang, E. I., \& Murray, N. 2009, ApJ, 693, 23

Pillitteri, I., Wolk, S. J., Cohen, O., \& Kashyap, V., et al.2010, ApJ, 722, 1216

Pillitteri, I., Wolk, S. J., Lopez-Santiago, J., \& Günther, H. M., et al.2014, ApJ, 785, 145

Pillitteri, I., May A., Micela, G., Sciortino, S., Wolk, S.J., Matsakos, T. 2015 ApJ, 805, 52

Poppenhaeger, K. \& Wolk, S. J. 2014, A\&A, 565, L1

Poppenhaeger, K., Schmitt, J. H. M. M., \& Wolk, S. J. 2013, ApJ, 773, 62

Poppenhaeger, K. \& Schmitt, J. H. M. M. 2011, ApJ, 735, 59

Vidal-Madjar, A., Lecavelier des Etangs, A., \& Désert, et al. 2003, Nature, 422, 143 